prediction is based must be made before, or at the outset of, treatment. Only one of the measures cited (the release of prolactin) can be made at the first ECT; the other measures can only be made once treatment is complete and cannot be predictive. Prediction of relapse after successful treatment is another important clinical concern, but one I did not cover in my review. Concerning the release of prolactin after ECT, I do not doubt that real ECT produces a greater increase than simulated ECT.

For reasons I have already given in the review, I cannot recommend any of these biological measures to clinicians to improve their ability to select depressed patients who will recover after ECT.

University Department of Psychiatry

Allan Scott

The Kennedy Tower

Royal Edinburgh Hospital

Morningside Park

Edinburgh EHIO 5HF

\section{EEG monitoring of ECT}

SiR: McCreadie et al (Journal, February 1989, 154, 229-231) propose a case for routine EEG monitoring of ECT, particularly for unilateral treatments. However, there are certain flaws in their paper.

Firstly, Dr McCreadie et al have misquoted the findings of Christensen \& Koldbaek (1982). They state that Christensen \& Koldbaek found $43 \%$ of fits inadequate in terms of EEG signs when judged by clinical observation alone. In fact, they reported the opposite: $43 \%$ of fits were inadequate as judged by the clinical method, but only $9 \%$ were inadequate by EEG criteria.

A further misconception concerns the ability of the EEG to demonstrate all seizure activity. Where disagreement arises between the EEG and clinical methods as to whether a seizure has occurred, Dr McCreadie et al unjustifiably assume that the EEG finding is correct and the clinical method false. One type of disagreement (no EEG fit/clinical fit) is interpreted as clinical misdiagnosis of a seizure. However, it is well recognised that seizure activity is not always visible even on a conventional multichannel EEG, let alone the single channel used by the authors. It is questionable whether two bifrontal EEG leads will reliably pick up seizure activity, as the potential difference between the EEG electrodes may be equal in a bilateral seizure. Furthermore, as seizure activity progresses through the cerebral hemispheres, the precise duration of a seizure cannot be demonstrated by a single channel EEG.
The authors state that if only EEG seizures lasting over 25 seconds are counted, disagreement between the EEG and clinical method increases. However, this remark is tautological, since clinical seizures were not measured. In fact, Dr McCreadie et al have shown that, as far as eliciting whether a seizure has occurred is concerned, the EEG and clinical methods agree in the vast majority of cases, with no significant difference between bilateral and unilateral treatments $(97.5 \%$ and $92 \%$ respectively). Thus the conclusion that unilateral ECT requires EEG monitoring seems unwarranted from their data.

Finally, I should like to contribute some points, derived from my own experience (Jones, 1988), to counterbalance the argument that EEG monitoring of ECT is clinically useful.

Firstly, reliable performance of EEG electrodes requires time-consuming preparation of the scalp. In practice, this means that the EEG electrodes and leads have to be attached to the patient prior to anaesthesia. In my experience patients, who are already apprehensive about ECT, find this procedure distressing.

Secondly, frontotemporal EEG electrodes can obstruct the correct application of the ECT electrodes.

Thirdly, mains-powered EEG machines which are not designed for use with ECT are potentially dangerous if so used. This is because, unless the mains connections are electrically isolated, there would be a possibility of the electroshock short-circuiting from a scalp electrode to earth through the EEG machine, which could result in electrocution of the patient. I overcame this problem by adapting a battery operated audible biofeedback machine for monitoring of ECT. Two of the more recently developed ECT devices, the Thymatron and the MECTRA, incorporate a single lead EEG and have circuitry to eliminate electrical errors of this kind.

Fourthly, few junior doctors administering ECT are proficient in reading the EEG. Moreover, preoccupation with the EEG display can detract from their attention to the clinical care of the patient.

Unfortunately, not all anaesthetists are aware of the importance of timing seizure duration in ECT, and therefore sometimes fail to adjust the dose of muscle relaxant during a course of ECT. However, with judicious use of anaesthetic agents, clinical monitoring of seizure duration is usually possible. The case for EEG monitoring of ECT remains unproven.

Department of Mental Health

ERICA M. Jones 
References

Christensen, P. \& Koldbaek, I. (1982) EEG monitored ECT. British Journal of Psychiatry, 141, 19-23.

JONES, E. (1988) Inadequate seizures with electroconvulsive therapy. British Journal of Psychiatry, 153, 264-265.

SIR: Dr Jones has suggested we have misquoted Christensen \& Koldbaek (1982). Not so. Perhaps the insertion of two commas might help her understand the sentence "... found $43 \%$ of fits inadequate, in terms of EEG signs, when judged by clinical observation alone".

It is widely accepted that EEG monitoring provides the most accurate measure of seizure activity. For better or worse, we used it as the standard by which we judged clinical recognition. Thus, to be consistent, no EEG fit/clinical fit is interpreted as clinical misdiagnosis of a seizure.

A tautology is "saying the same thing twice over in different words" (Oxford English Dictionary). We fail to see how changing the definition of an EEG seizure and looking again at clinical seizures is tautological.

Dr Jones has read correctly our paper when she says that in the vast majority of cases EEG and clinical methods do agree. Disagreement in $8 \%$ of unilateral fits suggests to her that EEG monitoring of unilateral ECT is unwarranted. That is debatable. Ten of 17 patients given unilateral ECT had at least one EEG monitored fit of less than 25 seconds. If fit length is therapeutically crucial, we repeat our suggestion that the case for routine EEG monitoring is then greatly strengthened.

Dr Jones has encountered practical difficulties in EEG monitoring. Such difficulties may exist, but can be overcome. ECT is an important treatment, and it behoves all of us to examine closely our clinical practice.

Department of Clinical Research

Robin G. MCCREADIE

Crichton Royal Hospital

Dumfries DG1 4TG

\section{Depression in attempted suicide}

SIR: Ennis et al (Journal, January 1989, 154, 41-47) state that our study of patients who had attempted suicide (Goldney et al, 1981) "reported the highest prevalence of depression". This is demonstrably not so, as two of the five other studies they quote in their Table II found an even higher prevalence of depression.

In considering the different sub-types of depression, there appear to be data missing from their Table I. Dr Ennis et al noted that our figure for the delineation of endogenous depression, $36 \%$, was higher than those for affective disorder or endogenous depression detected by others, which were of the order of $10-17 \%$. Their results are also appreciably greater than those previous results, but they have chosen to report them as indicating that "only" $31 \%$ were diagnosed with a major depressive episode.

They correctly acknowledge the problems of nosology of depression, and suggested that, "since only $8 \%$ of the sample met criteria for melancholia, bipolar illness or major depression with psychotic features, the rate of 'endogenous' depression can be assumed to have been low". This appears to be an unnecessarily restrictive assumption. Indeed, their figure of $31 \%$ is not dissimilar to the $36 \%$ of our subjects who were delineated as having "endogenous" depression, based on responses to the Levine-Pilowsky Depression (LPD) Questionnaire (Pilowsky \& Boulton, 1970). At the very least, it can be asserted that the symptoms which contributed to that allocation of diagnosis by the LPD, and which were described in an earlier Australian study (Goldney \& Pilowsky, 1979), are similar to those required for a DSM-III diagnosis of major depression.

The significance of individual symptoms in depression has long been debated. Pollitt (1971) postulated the concept of a "depressive functional shift" to describe the physiological symptoms which delineate depressive illness, and noted that it was an attempt "to find a nucleus of depressive illness; a timeless clinical index which, while being independent of culture and era, could be confidently assessed and communicated". He added that the value of the concept was that "a functional shift, however small, could be useful in distinguishing depressive illness from natural unhappiness". The symptoms employed by Dr Ennis et al to fulfil DSM-III criteria for a major depressive episode and those employed in our study to delineate endogenous depression are consistent with Pollitt's "depressive functional shift".

It is tempting to draw an analogy between angina and myocardial infarction, with the 'functional shift' of symptoms of depression being analogous to angina. Cardiologists have the benefit of electrocardiogram and enzyme changes to delineate the boundary between angina and infarction; psychiatrists as yet have no such instruments to provide precise delineation between the symptoms of the 'functional shift' and a depressive illness.

The above points are somewhat speculative. What is not speculative is the manner in which Dr Ennis et al have reported that "only" $31 \%$ of their subjects were diagnosed as having a major depressive episode, despite their figure being higher than a number of 